$$
\begin{gathered}
\text { CONF- } 9406286-1 \\
\text { UCRL-JC-118849 } \\
\text { PREPRINT }
\end{gathered}
$$

\title{
Application of Spectral Line Shapes to the Study of High Density ICF Plasmas
}

C. J. Keane, B. A. Hammel, S. H. Langer, R. W. Lee, A. Calisti, L. Godbert, R. Stamm, and B. Talin

This paper was prepared for submittal to the 12th International Conference on Spectral Line Shapes Toronto, Canada June 13-17, 1994

September 1, 1994

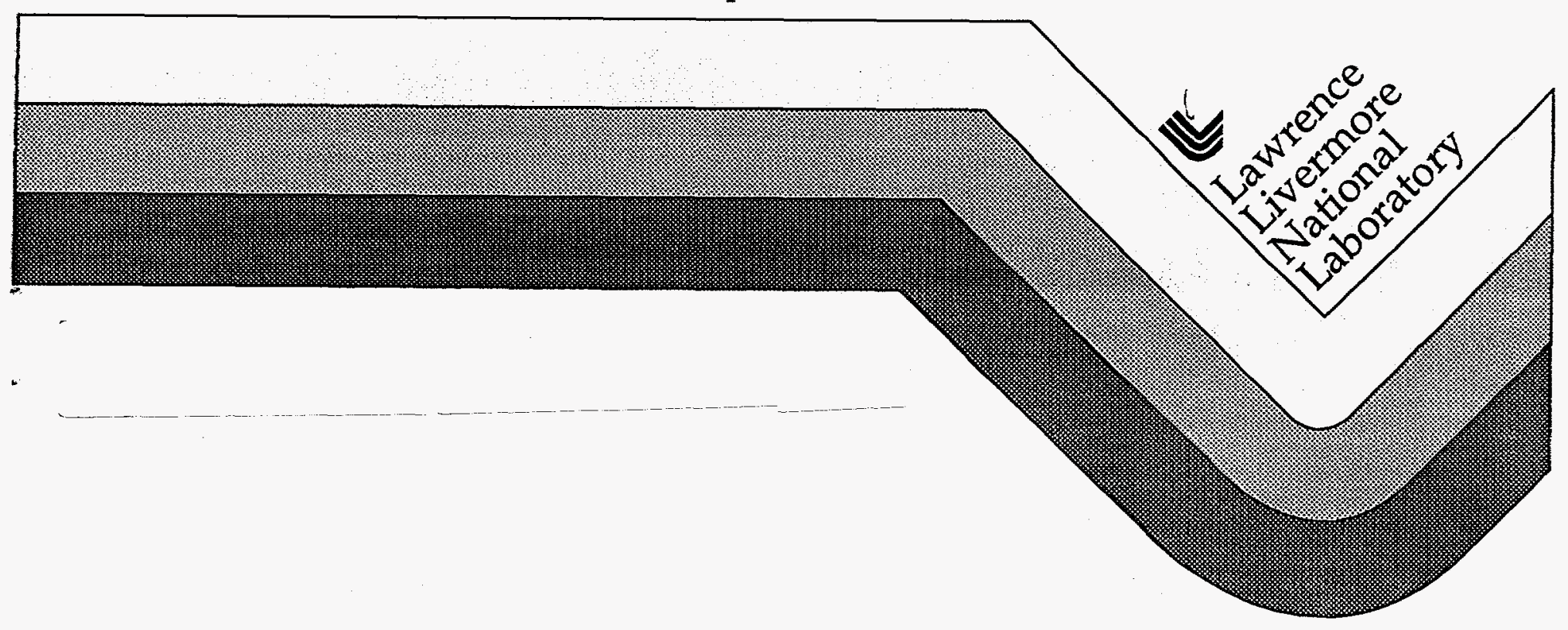




\section{DISCLAIMER}

This document was prepared as an account of work sponsored by an agency of the United States Government. Neither the United States Government nor the University of California nor any of their employees, makes any warranty, express or implied, or assumes any legal liability or responsibility for the accuracy, completeness, or usefulness of any information, apparatus, product, or process disclosed, or represents that its use would not infringe privately owned rights. Reference herein to any specific commercial product, process, or service by trade name, trademark, manufacturer, or otherwise, does not necessarily constitute or imply its endorsement, recommendation, or favoring by the United States Government or the University of California. The views and opinions of authors expressed herein do not necessarily state or reflect those of the United States Government or the University of California, and shall not be used for advertising or product endorsement purposes. 


\section{DISCLAIMER}

Portions of this document may be illegible in electronic image products. Images are produced from the best available original document. 


\title{
Application of Spectral Line Shapes to the Study of High Density ICF Plasmas
}

\author{
C.J. Keane, B.A. Hammel, S.H. Langer, and R.W. Lee \\ Lawrence Livermore National Laboratory, Livermore, CA 94550 \\ A. Calisti, L. Godbert, R. Stamm, and B. Talin \\ Universite de Provence, Centre St. Jerome, Marseille, France
}

\begin{abstract}
Spectral line broadening manifests itself in the study of high density inertial confinement fusion (ICF) plasmas in two important ways. First, comparison between measured and calculated lineshapes of individual spectral lines or groups of lines is used to diagnose plasma conditions in dense ICF plasmas, particularly in implosions. Secondly, through the emission and absorption coefficients spectral lineshapes serve as important inputs to plasma spectroscopy simulation codes which calculate simulated spectra from ICF targets. We discuss recent results from each of these areas. With regard to lineshape diagnostics, the advent of generalized line broadening codes has allowed the line profiles of complex multielectron emitters to be considered for diagnostic purposes. Particular example of this is the use of Ar He- $\beta$ and its associated dielectronic satellites as a diagnostic of $\mathrm{T}_{e}$ and $\mathrm{N}_{e}$, as well as the development of Ne-like Xe line broadening as a density diagnostic. With respect to simulation codes, the implementation of detailed lineshapes in calculations of this type is in many ways in its infancy. We present here examples of cases where effects related to spectral lineshapes such as continuum lowering and line transfer of Stark broadened lines are important so as to provide a stimulus for future work in this field.
\end{abstract}

\section{INTRODUCTION}

$X$-ray spectral diagnostics been extensively used as a diagnostic of density and temperature in a wide variety of plasmas relevant to inertial confinement fusion (ICF).1-11 In the case of diagnostics of imploding capsules, trace concentrations (typically at the fraction of a percent level) of mid-Z (Z 13-20) elements such as $\mathrm{Ar}$ or $\mathrm{Cl}$ are placed in the fuel and/or ablator regions iof the target. X-ray emission spectra from these dopants are used to diagnose plasma conditions in the capsule. The low concentration of these dopants results in minimal 
perturbation to the target hydrodynamics while at the same time yielding measurable $x$-ray spectra. Because such dopants may be locally placed (for example, in the gaseous fuel) this diagnostic information is intrinsically spatially resolved. Through the use of streak cameras or other techniques temporal resolution on a sub-100 ps time scale may be obtained as well. Thus, space and time resolved diagnostic information may be obtained.

X-ray spectra from these dopants typically yield a wealth of information regarding the density and temperature conditions present in the ICF target under consideration. In the case of simple, few electron emitters quantities such as ratios of individual lines, the inferred ionization balance, and individual line profiles provide diagnostic information. In the simple emitter case resonance line emission from the $\mathrm{H}$ - and He-like stages of the dopant (as well as associated dielectronic satellites) are typically observed.1-8,10 For the case where more complex emitters are present ratios of groups of lines are typically used for diagnostics. 9 As has been discussed elsewhere proper interpretation of measured line ratios often implies consideration of detailed spectral simulations of these targets in order to include line transfer and other dense plasma effects.

Spectral lineshape information enters into diagnostics of this type in two ways. First, the detailed lineshape of certain lines may serve as a diagnostic of density and/or temperature. In this case a single, optically thin line is usually considered so that kinetics, opacity and other effects usually considered in spectral simulations are unimportant. An example of this is Stark broadening of $\mathrm{H}$ - and He-like ion dopant lines which has been extensively used as a diagnostic of $\mathrm{Ne}^{1-11}$

The second area in which spectral lineshapes enter into x-ray spectral diagnostics of ICF plasmas is through spectral simulation codes.12-17 In these calculations the coupled kinetics and transfer equations are solved to produce emissivities and opacities as a function of space and time throughout the target. These are then used to produce a simulated spectra. Lineshapes enter here through the calculation of the plasma emissivity and opacity. An example of where lineshape effects may be important in this case is Ar spectra from indirectly driven implosions. ${ }^{5-9}$ In this case, proper calculation of the simulated spectra requires consideration of line transfer for the computed the optically thick $\mathrm{Ar} \mathrm{He}-\alpha$ and $\mathrm{Ly}-\alpha$ lines. As these lines are Stark broadened the line broadening treatment used in principle can effect simulated 
quantities of diagnostic importance such as the ratio of Ar Ly- $\beta$ to $\mathrm{Ar}$ He- $\beta$.

In this paper we consider recent results from these two areas. With respect to detailed lineshape calculations, we show how recent advances in the development of generalized lineshape codes have allowed us to consider more complex lineshapes for diagnostic purposes. In the area of spectral simulation, we show several cases where lineshape related effects are important. In fact, the inclusion of detailed lineshapes in spectral simulation codes is in its infancy, and a goal of this paper is to motivate further work in this area.

\section{ADVANCES IN THE USE OF COMPLEX LINESHAPES AS TEMPERATURE AND DENSITY DIAGNOSTICS}

Lineshapes of $\mathrm{H}$ - and He-like emitters have been used for some time as diagnostics of density in the fuel region of ICF implosions.19,11 Typically, resonance lines of $\mathrm{H}$ - and He-like emitters are used. The most recent examples of this involve the use of $\mathrm{Ar} \mathrm{He}-\beta$ and Ly- $\beta$ as density diagnostics in indirectly driven implosions. While this is often adequate for rough estimates, in recent indirect drive work it has been necessary to increase the range of lineshapes considered for several reasons. First, satellite lines to $\mathrm{Ar} \mathrm{He}-\beta$ have been observed to significantly affect the intensity of the Ar He- $\beta$ feature. ${ }^{5-9}$ Hence, it is desirable to calculate the line profile of $\mathrm{Ar} \mathrm{He}-\beta$ and its satellites self consistently. Secondly, some higher performance implosions currently done on Nova have pusher opacities sufficiently high so as to attenuate $\mathrm{Ar} \beta$ line emission at $3.5-4 \mathrm{keV} .5,19$ We are interested in developing line broadening diagnostics operating at higher photon energy. An obvious candidate for this is Ne-like Xe. This makes sense for two reasons. First, Xe gas is easily placed in the fuel region of Nova capsules. Secondly, Ne-like Xe 3-2 and 4-2- emission falls at roughly 4.5 and $6 \mathrm{keV}$, respectively. The 4-2 emission thus has particular promise as a density diagnostic.

Consideration of both of these problems requires consideration of lineshapes more complex than simple $\mathrm{H}$ - and He-like line profiles. Indeed, the tools to calculate such lineshapes have recently been written. Specifically, two codes, TOTAL ${ }^{20}$ and MERL, 21,22 are now available. Both codes take as input an atomic data set consisting of energy levels, statistical weights, and reduced matrix elements for an arbitrary emitter. Lineshapes are then calculated by working in the "standard" quasistatic ion/impact electron limit. Ion motion effects are 
currently being incorporated into each. The development of these codes has been crucial to the advancement of the field of diagnostic lineshapes for ICF. Results obtained with these codes for the Ar He$\beta /$ satellite and Ne-like Xe problems have been presented elsewhere;4-9 we briefly summarize the major results here.

We start by showing a typical spectrum from an indirectly driven ICF implosion in Fig. 1. The features seen here are common to all $\mathrm{Ar}$ spectra observed from indirectly driven implosions performed with either shaped or square pulses. The broadened Ar He- $\beta$ and Ly- $\beta$ lines

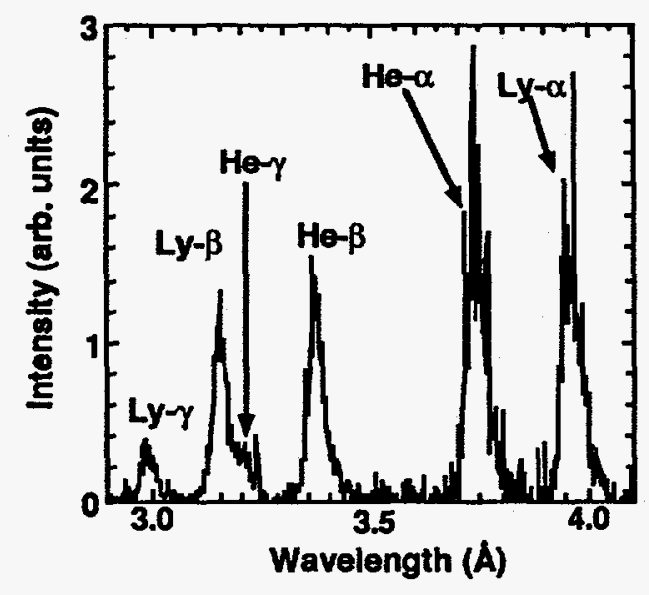

Figure1. Typical spectrum at peak emission time for an Ar doped, indirectly driven implosion.

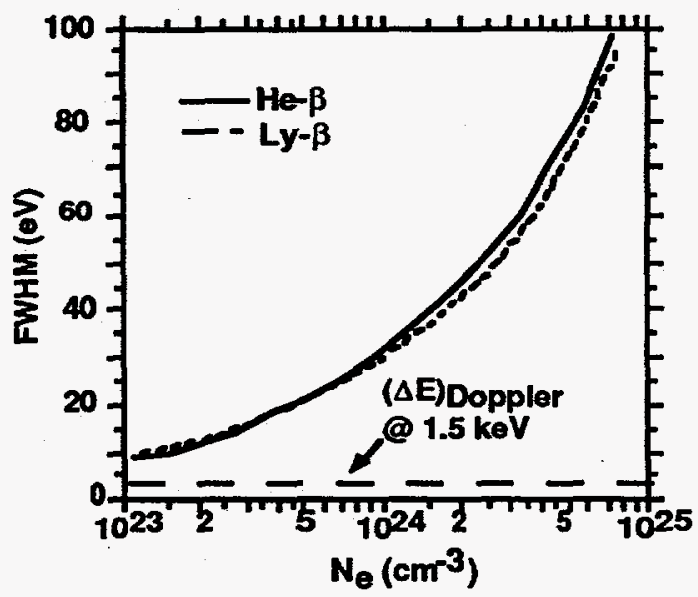

Figure 2. Width of $\mathrm{H}$ - and He-like $A r$ 3-1 lines vs. electron density for an electron temperature of $1 \mathrm{keV}$.

are apparent. (As discussed elsewhere, the ratio of Ly- $\beta$ to He- $\beta^{5-9}$ serves as a measure of spatially averaged fuel electron temperature.) The lineshapes shown in Fig. 1 have widths of order $30 \mathrm{eV}$ which is much greater than the Doppler width of about $1.5 \mathrm{eV}$, and are easily resolvable with current streaked spectrographs. The width of the Ar He- $\beta$ and Ly- $\beta$ lines thus provides a density diagnostic.

The density dependence of these linewidths as calculated using a simple K- shell quasistatic ion/impact electron treatment is shown in Fig. 2. It is clear that for conditions typical of current implosions $\left(\mathrm{Ne}_{\mathrm{e}}{ }^{20^{24} \mathrm{~cm}^{-3}}\right.$ ), Stark broadening dominates and the linewidth is a useful electron density diagnostic.

Things become more complicated, however, when Li-like satellites to Ar He- $\beta$ are considered. These satellites are commonly seen in Nova implosion spectra ${ }^{5-9}$ and can be seen at a fairly low level in Fig. 1. 
Transitions of the type $1 \mathrm{~s}^{2} \mathrm{nl}-1 \mathrm{snl} 3 \mathrm{l}^{\prime}$ lie just on the long wavelength side of the Ar He- $\beta$ line and potentially can affect the electron density inferred from Stark broadening of Ar He- $\beta$. In order to consider this more quantitatively detailed line shape calculations including both Ar $\mathrm{He}-\beta$ and its dielectronic satellites have been carried out using both TOTAL ${ }^{8-10}$ and MERL. ${ }^{18}$ Some comparisons between these two codes for this case are presented elsewhere. ${ }^{7}$ In Fig. 3 TOTAL computed lineshapes of the Ar He- $\beta$ line and dielectronic satellites are shown. TOTAL takes in as input the complete energy level structure of the Helike $\mathrm{Ar} n=3$ manifold as well as the associated Li-like Ar autoionizing levels. Mixing of these levels through the ion microfield as well as electron broadening occurring through collisions is considered. To date, the $2131^{\prime}$ and $3131^{\prime}$ Li-like autoionizing levels have been considered; efforts are underway to include the effects of 4131' autoionizing levels. ${ }^{23}$ The full non-LTE populations of these excited states are used. It should be emphasized that TOTAL solves the quasistatic line broadening problem for all of the relevant levels

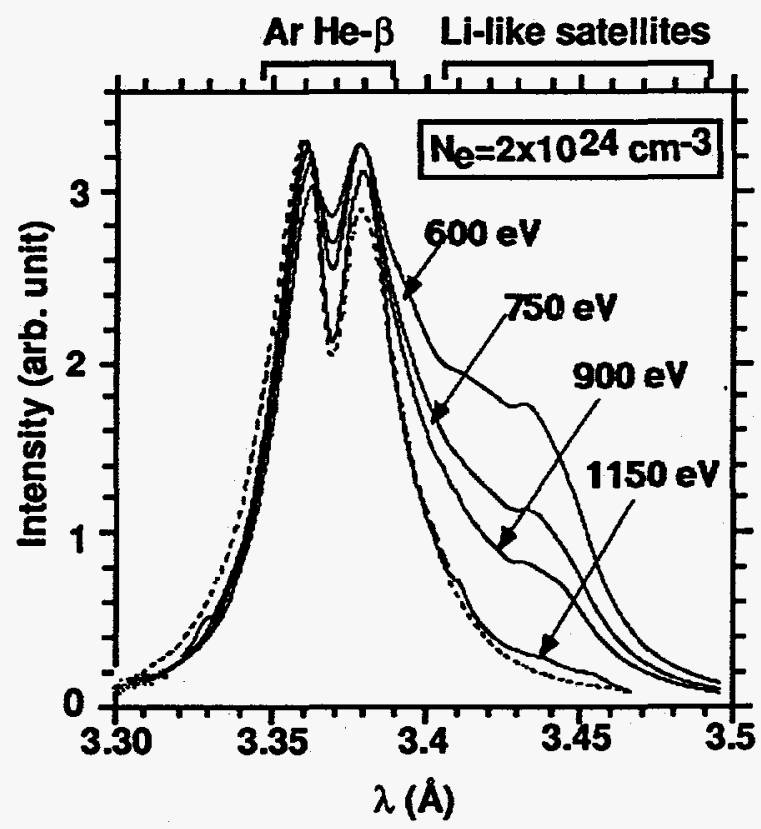

Figure 3. TOTAL computed profile of He-like Ar 3-1 emission along including associated dielectronic satellites.

Results shown for electron temperature of $1150 \mathrm{eV}$. Dash: He-like Ar 3-1 profile only at $1150 \mathrm{eV}$. simultaneously. As a final point, variant of MERL has been developed which includes ion motion effects. This code is currently being used to study ion motion effects on the combined profile of $\mathrm{Ar} \mathrm{He}-\beta$ and its dielectronic satellites. ${ }^{24}$

Figure 3 shows that satellite emission can strongly modify the observed profile, especially at low temperatures and high densities. The result of this is that the combined $\mathrm{He}-$ $\beta /$ satellite lineshape provides a simultaneous electron density and electron temperature diagnostic. In particular, the width of the entire feature and the intensity of the satellite contribution increase with $\mathrm{Ne}_{e}$ and decrease with $\mathrm{T}_{e}$, respectively. 
From Fig. 3 it is evident that fitting experimentally measured He$\beta /$ satellite line profiles to theory yields a best fit for a given value of $\mathrm{Ne}_{e}$ and $\mathrm{T}_{\mathrm{e}}$ and thus a simultaneous diagnostic of these quantities. Figure 4 shows an example of this procedure for 1-ns square drive and pulseshaped drive Nova implosions. In this figure measured He- $\beta$ /satellite
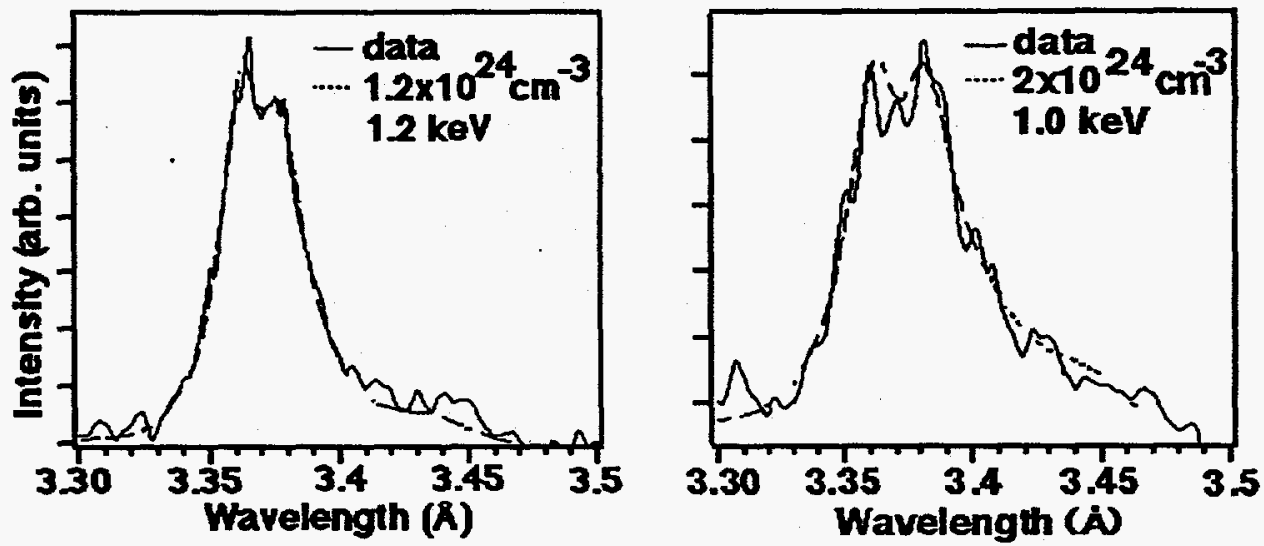

Figure 4. Measured He-like Ar 3-1 and satellite lineshapes along with best fit calculated line profile. Left: results for 1-ns square laser pulse implosion. Right: results for 3:1 contrast laser pulse implosion.

profiles are fit to TOTAL calculated lineshapes. The latter include contributions from the $\mathrm{He}-\beta$ line as well as the $213 \mathrm{l}^{\prime}$ and $313 \mathrm{l}^{\prime}$ autoionizing levels. The best fits yield inferred spatially averaged plasma conditions of $\left(\mathrm{N}_{\mathrm{e}}, \mathrm{T}_{\mathrm{e}}\right)=\left(1.2 \times 10^{24} \mathrm{~cm}^{-3}, 1.2 \mathrm{keV}\right)$ and $\left(2.0 \times 10^{24}\right.$ $\mathrm{cm}^{-3}, 1.0 \mathrm{keV}$ ) for the 1-ns square and shaped drive cases respectively. This is in accord with expectations; the shaped pulse results in a more isentropic implosion and thus a higher fuel density.

Figures 3 and 4 demonstrate the importance of generalized line broadening codes such as TOTAL and MERL in generating line profiles for complex emitters for purposes of fitting to data. TOTAL is also being used to develop line broadening of Ne-like $\mathrm{Xe}$ as a density diagnostic for use in high performance implosions characterized by higher fuel densities and pusher opacities compared to those typical of the targets shown in Fig. 1-4.5,19 Initial results for Ne-like Xe lineshapes assuming static ion and impact electron broadening have been described previously. ${ }^{4}$ More recently, the TOTAL code has been extended to include ion motion effects, as described in detail elsewhere. 25 The method used to include ion motion effects assumes that the dynamic 
lineshape results from a stationary Markovian process induced by the fluctuating ion microfield, which mixes together the static components. ${ }^{25}$ This method differs from those which start with a mathematically modeled stochastic microfield.27,28 The TOTAL method requires an estimate of the fluctuation frequency for the

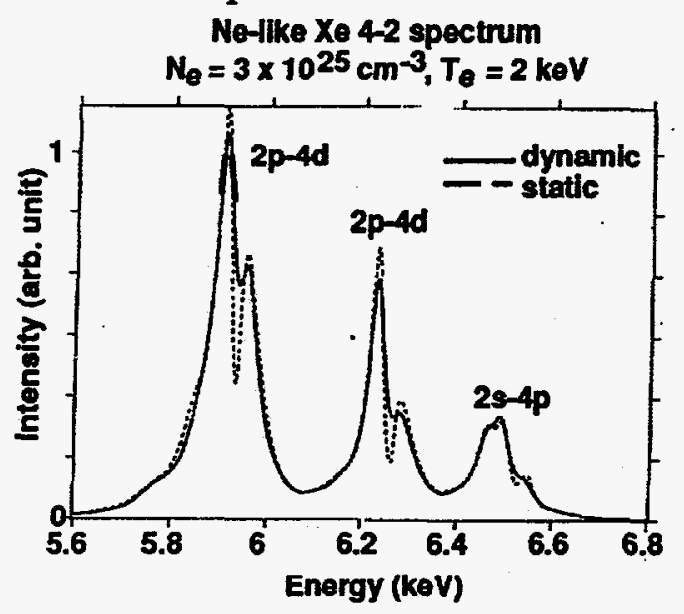
microfield, is required, which is usually obtained from molecular dynamics simulations.

Detailed calculations of Ne-like Xe lineshapes including the effects of ion motion have been carried out and will be presented elsewhere; 26 here we show a selection of recent results. First, we recall that as discussed in Ref. 4 the 4-2 lines in Ne-like Xe show greater promise than the 3-2 lines in terms of Figure 5. TOTAL calculated lineshapes linewidth sensitivity to density. for Ne-like Xe 4-2 lines showing effects of This is because the 3-2 transitions ion dynamics. show relatively little pressure broadening in the range $10^{24} \mathrm{~cm}^{-3}<\mathrm{N}_{e}<3 \times 10^{25} \mathrm{~cm}^{-3}$. Hence, most recent calculations assessing ion motion effects have focused on the 4-2 and 5-2 transitions, although 3-2 line profiles computed with ion dynamics are also under investigation. Figure 5 shows TOTAL computed lineshapes for the 4-2 transitions in Ne-like $X e$ at $\mathrm{N}_{e}=3 \times 10^{25}$ $\mathrm{cm}^{-3}$ and $\mathrm{T}_{\mathrm{e}}=2 \mathrm{keV}$. Results are shown for both static and dynamic ion broadening. The inclusion of ion motion is seen to fill in "dips" near line center, as would be expected. The relatively broad width of the 4-2 lines suggests they hold promise as density diagnostics.

The 5-2 lines are also potentially useful as diagnostics as can be seen from Fig. 6, which plots the width of the 2p-5d transitions vs. $\mathrm{Ne}_{\mathrm{e}}$. Other factors must be considered when examining the $2 p-5 d$ lines as a density diagnostic, however. 


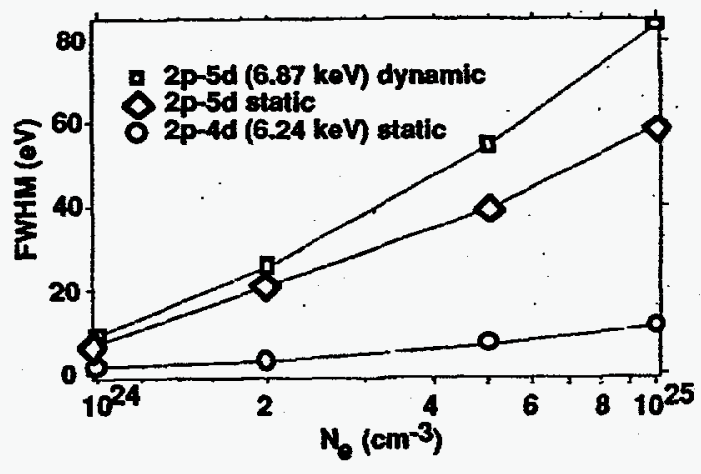

Figure 6. FWHM of Ne-like $X e$ 2p-4d and $2 p-5 d$ lines vs. electron density.
First, the 2p-5d lines should be somewhat weaker than the $2 p-$ $4 d$ transitions. Secondly, the presence of satellite lines may make measurement of linewidths using all of these transitions difficult. Nearby satellites do not appear to be a problem for $n=3-2$ and 4-2 transitions, but it has not be considered as of yet for 5-2 lines. Finally, continuum lowering affects must be assessed. Simple estimates based on the Stewart-

Pyatt formula show that the continuum is lowered to the $n=5$ and $n=4$ levels at $\mathrm{N}_{\mathrm{e}}=10^{25} \mathrm{~cm}^{-3}$ and $\mathrm{Ne}_{\mathrm{e}}=3 \times 10^{25} \mathrm{~cm}^{-3}$, respectively. These densities thus are a rough upper limit for the region of applicability of pressure broadening of these lines for density diagnostics.

\section{LINESHAPES IN SPECTRAL SIMULATION CODES}

In terms of the number and variety of lineshape calculations required, spectral simulation codes are by far the largest "user" of line profile information in the plasma spectroscopy community. By "spectral simulation" code we mean here a code that reads in hydrodynamic information from a simulation code, experiment, or elsewhere, and solves the coupled kinetics and transfer equations to produce level populations as a function of space and possibly time. These level populations are then used to construct time and space dependent emission and absorption coefficients. A simulated spectra as seen by an observer along any given line of sight is then generated by formally solving the equation of radiative transfer along the desired ray path. Codes of this type have been heavily used by the ICF, 5-11 magnetic fusion, ${ }^{29}$ x-ray laser, 30 and short-pulse laser-produced plasma communities, 14 among others.

Spectral lineshape information plays an important role in codes of this type because it enters directly into the calculation through the emission and absorption coefficients $\varepsilon_{v}$ and $\kappa_{v}$. There are in fact two important ways in which variations in $\varepsilon_{v}$ and $\kappa_{v}$ arising from line profile variations can affect simulated spectra. First, variation in the line absorption profile can directly result in changes in opacity throughout 
the region of interest. For cases where opacity is important in driving the plasma kinetics, this can obviously lead to different answers for the computed level populations and thus the computed spectra. Secondly, assuming the kinetics is known, simply varying the lineshapes used in the formal solution of the transfer equation can changes items of diagnostic interest such as the peak to peak ratio of different lines. Given that in experimentally "noisy" situations one can often only measure peak-to peak line ratios, lineshapes can be critical to data interpretation. All of these effects are magnified many times for complex emitters where many overlapping lines are present.

In addition to the roles of spectral lineshapes themselves, there are two related theoretical problems of significance to dense plasma spectral simulation, namely the natural line width and continuum lowering. 15,31 The first of these refers to the natural linewidth in dense plasmas increasing to very large values as one proceeds to higher density and higher principal quantum numbers. Simply put, at high densities inelastic electron collisions (electron impact excitation, deexcitation, and ionization) can lead to significant natural broadening. As an example, for the $\mathrm{H}$-like $\mathrm{Ar} \mathrm{n}=3$ level at $\mathrm{Ne}_{\mathrm{e}}=10^{24} \mathrm{~cm}^{-3}$ and $\mathrm{T}_{\mathrm{e}}=1$ $\mathrm{keV}$, the time for electron impact excitation from $n=3$ to $n=4$ corresponds to a natural width of about $10 \mathrm{eV}$, which is $30 \%$ of the 3-1 static ion width! Other cases of seemingly greater importance can be found by considering more complex emitters where certain states of importance for line broadening may have levels very close by in energy to which inelastic collisions may occur very rapidly. Some of this problem may in principle by mitigated by continuum lowering. As discussed in Ref. 15, the interplay between continuum lowering and natural linewidth can be attacked by resorting to an impact approximation type calculation. This has not been carried out as of yet but it is very high priority. Other ideas for treating this problem have also been considered informally but more work is required. ${ }^{32}$

In an ideal world one would like detailed line-shape codes to be able to be run inline and completely self-consistently with kinetics and line transfer calculations, including the effects of continuum lowering and natural linewidth. Clearly, this goal has not been obtained as of yet. Solution of this problem is one of the major challenges facing plasma spectroscopy. Obviously we cannot present a solution of this problem here. Rather, we will present several ICF situations where lineshapes and related physics such as continuum lowering play an important role in spectral simulation. We will then discuss existing spectral 
simulation codes and their methods for treating physics related to spectral lineshapes.
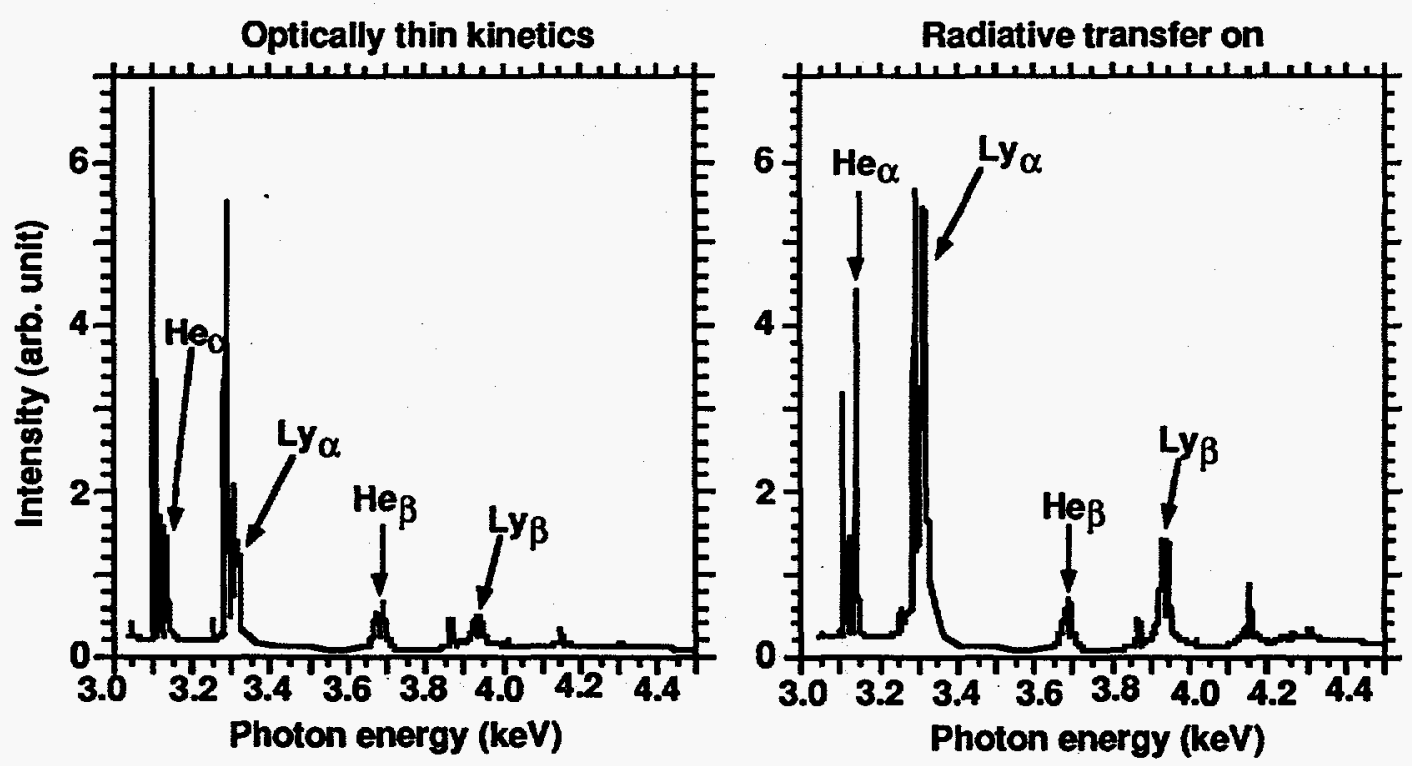

Figure 7. Simulated Ar spectra from an indirectly driven implosion calculated using the DSP postprocessing code. Left: Optically thin kinetics. Right: line photon field considered in kinetics calculation.

The first ICF situation which demonstrates the importance of lineshapes in shown in Fig. 7, which shows calculated spectra from the simulation of an Ar doped indirectly driven implosion of a DD filled plastic capsule. These spectra were calculated by postprocessing LASNEX ${ }^{33}$ hydrodynamics output with the DSP ${ }^{12}$ spectral postprocessing code. Spectra calculated with and without including line transfer in the kinetics calculations are shown. More specifically, in the no transfer calculation, the Ar level populations are solved using the local values of $\mathrm{T}_{e}, \mathrm{Ne}_{e}$, and $\mathrm{N}_{\mathrm{i}}$ only. In the calculation including radiative transfer all lines shown in the spectrum are transferred; the line intensities and level populations are computed self consistently. Opacity is only significant for the He- $\alpha$ and Ly- $\alpha$ lines, however; these lines have an optical depth of order 10.

As discussed in detail in Ref. 9, two features regarding the effects of transfer are immediately apparent. First, the intensity of the $\alpha$ lines is increased. Secondly, the ratio of the optically thin $\mathrm{He}-\beta$ and $\mathrm{Ly}-\beta$ lines of $\mathrm{Ar}$ is varied. The latter occurs through an increase in the population of $\mathrm{H}$-like Ar throughout the target when line transfer is considered.9 ${ }^{9}$ This 

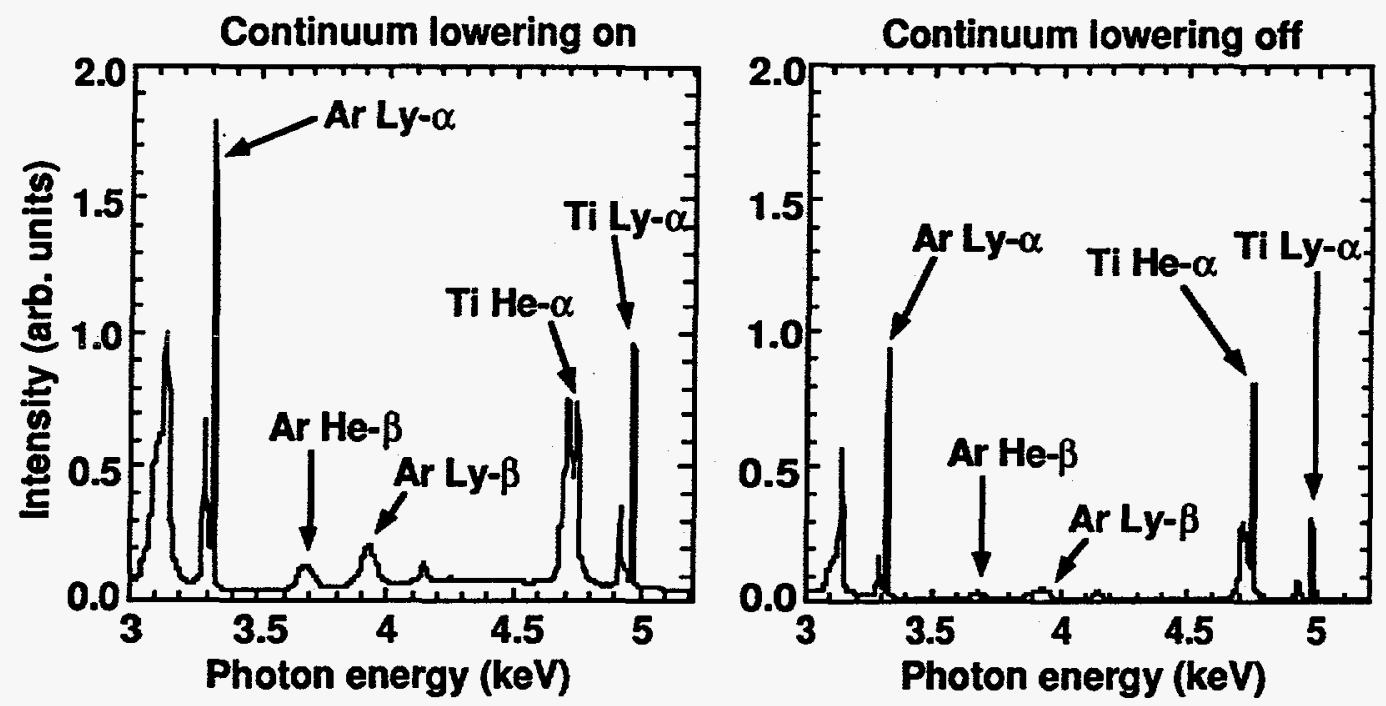

Figure 8. Computed Ar/Ti spectrum from an implosion with Ar doped into the fuel and $T i$ doped into the innermost $3-\mu \mathrm{m}$ of plastic adjancent to the fuel. Left: Continuum lowering included. Right: Continuum lowering neglected.

is obviously important as the ratio of these two lines serves as a diagnostic of spatially averaged fuel electron temperature. Indeed, obtaining any kind of reasonable agreement with experiment requires that these transfer effects be considered.8,9 Figure 7 thus represents a case where line transfer of Stark broadened lines play a crucial role in modelling experimental data. As such experiments of this type form a testbed for tests of combined lineshape/radiative transfer effects.

Figure 8 shows a second example from ICF implosions demonstrating the importance of continuum lowering in the computation of simulated spectra. (The spectra shown in Figs. 8 and 9 were generated by postprocessing LASNEX ${ }^{33}$ hydrodynamic code output with the CRETIN 13 code.) The spectra shown in Fig. 8 is from the simulation of a capsule with dopants placed so as to diagnose fuel/pusher mix.19,34 In this case the fuel is doped with Ar as previously. In addition, the innermost $3-\mu \mathrm{m}$ of pusher closest to the fuel region is doped with $\mathrm{Ti}$ at the $0.07 \%$ atomic level. In experiments using these dopants the degree of pusher/fuel mix is varied experimentally by varying the size of surface perturbations placed on the outer capsule surface. ${ }^{34}$ The increasing degree of mix associated with rougher capsules implies that more of the pusher material is mixed into the fuel. Thus, as the degree of pusher/fuel mix increases, one expects the ratio of $\mathrm{Ti}$ to Ar emission to increase. ${ }^{19}$ In Fig. 8 the computed spectrum at peak emission for a $\mathrm{Ar} / \mathrm{Ti}$ doped capsule is shown with and without continuum lowering 
considered. The continuum lowering model used is a modified version ${ }^{31}$ of the Stewart/Pyatt formalism and is thus quite a simple treatment. Note the sensitivity of the ratio of Ti Ar He- $\alpha /$ Ar Ly- $\beta$. This ratio has been proposed as a measure of pusher/fuel mix. The variation in this ratio as a function of continuum lowering implies that a more careful treatment of this subject in the context of spectral simulation codes is required.

The last example showing the importance of lineshapes to ICF spectral simulation codes is presented in Fig. 9. This is another "mix diagnostic" calculation of the type shown in Fig. 8, with the exception that the $\mathrm{Ar}$ and $\mathrm{Ti}$ dopants have been replaced by $\mathrm{Xe}$ and $\mathrm{Cr}$,
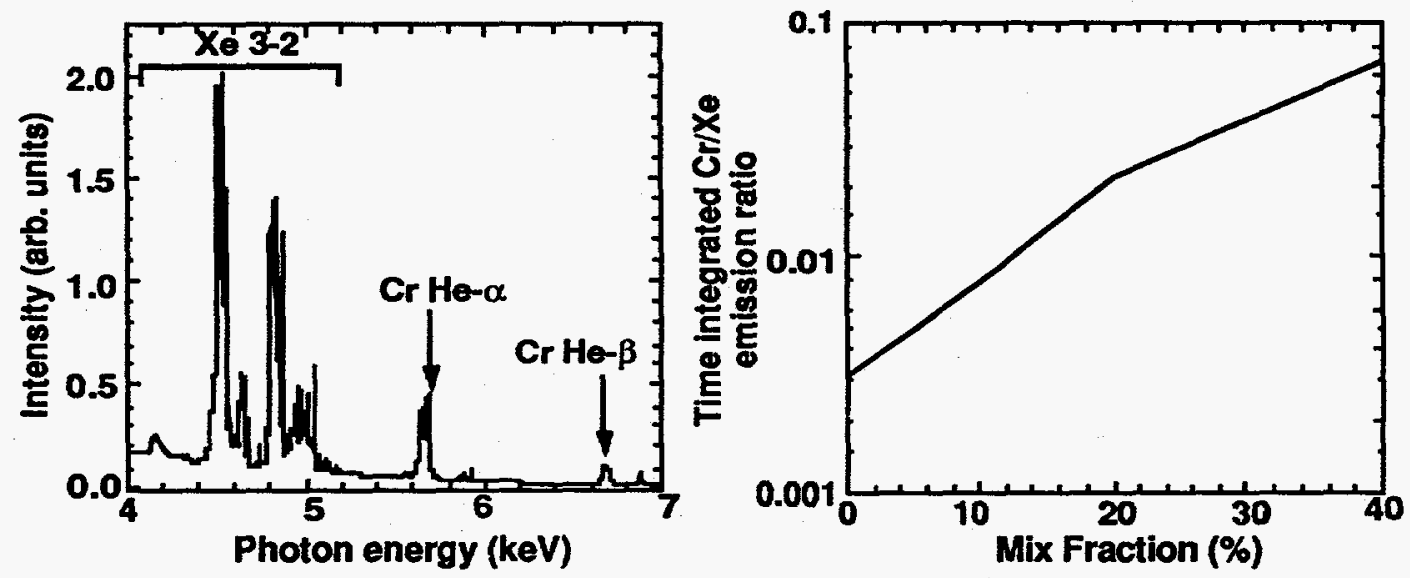

Figure 9. Computed spectrum from and indirectly driven implosion with $X e$ seeded into the fuel and $C r$ seeded into the innermost 3- $\mu \mathrm{m}$ of plastic closest to the fuel. Left: Computed spectru $m$ at time of peak emission. Right: Time integrated ratio of He-like $\mathrm{Cr}$ 2-1 emission to Ne-like Xe 3-2 region emission plotted vs. mix fraction.

respectively. Figure 9 shows a calculated spectrum at time of peak emission for such a case, as well as the behavior of the ratio of $\mathrm{Cr}$ to $\mathrm{Xe}$ emission as a function of mix fraction. (The "mix fraction" is approximately the extent of the mixed region at the fuel/pusher interface expressed as a function of the fuel radius.) In this case the $\mathrm{Cr}$ He- $\alpha$ line is optically thick, as are the 3-2 Ne-like Xe resonance lines. These lines have been transferred in the calculation shown in Fig. 9, and hence the assumed lineshapes for these transitions enter into the kinetics calculation. The remainder of the spectrum consists of many hundreds of overlapping satellite lines. All lines considered in this problem are treated with simple Voigt profiles with an ad-hoc prescription for Stark broadening included in the Lorentzian portion of the lineshape. This is clearly a very crude treatment for this case 
with its many overlapping lines. (Simple estimates indicate that for this case electron impact broadening is greater than ion quasistatic effects and is of order or slightly greater than Doppler broadening.) Figure 9 thus represents a good example of a "benchmark" case where hundreds of detailed lineshapes are needed at a given time step.

We conclude this section by reviewing examples of available plasma simulation codes and how they treat the physics described here. Table I compares some major characteristics of existing spectral simulation codes. The "lineshapes (kinetics)" heading refers to the method by which lineshapes are incorporated into the line transfer. In a similar vein "lineshapes (spectrum)" refers to how lineshapes are used in the generation of the emission and absorption coefficients given the level populations. The "level structure" column indicates how the level structure is modified in the kinetics to account for plasma microfields. (This is not generally done in existing simulation codes.)

\section{Table I. Spectral Simulation Code Characteristics}

\begin{tabular}{|c|c|c|c|c|}
\hline $\begin{array}{l}\text { Code } \\
\text { Type }\end{array}$ & $\begin{array}{l}\text { Continuum } \\
\text { Lowering }\end{array}$ & $\begin{array}{l}\text { Lineshapes } \\
\text { (kinetics) }\end{array}$ & $\begin{array}{l}\text { Level } \\
\text { Structure }\end{array}$ & $\begin{array}{l}\text { Lineshapes } \\
\text { (spectrum) }\end{array}$ \\
\hline 1 & None & None & Zero field & Doppler \\
\hline 2 & Stewart-Pyatt & $\begin{array}{l}\text { Voigt w/ } \\
\text { crude Stark } \\
\text { estimate in } \\
\text { Lorentzian }\end{array}$ & Zero field & As kinetics \\
\hline $2 \mathrm{~A}$ & Stewart-Pyatt & $\begin{array}{l}\text { Voigt w/ } \\
\text { crude Stark } \\
\text { estimate in } \\
\text { Lorentzian }\end{array}$ & Zero field & $\begin{array}{l}\text { Full inline } \\
\text { K-shell Starl } \\
\text { profiles }\end{array}$ \\
\hline 3 & Stewart-Pyatt & $\begin{array}{l}\text { Inline K-shell } \\
\text { Stark }\end{array}$ & Zero field & As kinetics \\
\hline 4 & Stewart-Pyatt & $\begin{array}{l}\text { Detailed } \\
\text { profiles split } \\
\text { up as in levels }\end{array}$ & Zero field & As kinetics \\
\hline 5 & Self consistent & Self consistent & $\begin{array}{l}\text { Self } \\
\text { consistent }\end{array}$ & As kinetics \\
\hline
\end{tabular}


The DSP code used in Fig. 7 is a code of type " $2 A^{\prime \prime}$ above. DSP12 uses a simplified form of solution of the coupled transfer/kinetics equations. The CRETIN ${ }^{13}$ code used in Figs. 8-9 is of type 2 above. It has a simpler lineshape treatment than DSP but includes a more sophisticated complete linearization radiative transfer package. Most codes in existence fall into categories 1-3. A code along the lines of type 4 above has been developed 16 and is being extended to handle dense plasma phenomena necessary for ICF plasma modeling.

Note that we have not considered different line transfer models as a separate category above. This is because by and large most ICF line transfer problems are not difficult, assuming a given (usaully simple) lineshape prescription. That is, the solution of the coupled transfer and kinetics equations is not especially difficult due to the relatively modest optical depths and minimal photon scattering present. (The latter is equivalent to stating that the probability of photon destruction is generally high due to high rates of collisional deexcitation between excited states.) The difficult part of the line transfer problem is in defining the associated kinetics and line broadening problems so as to be self consistent. 15

Indeed, a "type 5" code as defined above represents the "ultimate" simulation capability in that line broadening, continuum lowering, and plasma microfield effects on kinetics are handled self consistently. (It is clearly a long way from step 4 to step 5.) These phenomena are strongly coupled in dense plasmas and an adequate, self consistent treatment is a major goal for plasma spectroscopy research.

The main point of Table $I$, then, is that the treatments of continuum lowering, Stark broadening, and plasma microfield dependent kinetics in plasma simulation codes are quite crude to date. In particular, as shown in this paper, assumptions regarding lineshapes and continuum lowering affect simulated spectra from ICF targets. There is much room for improvement of models of dense plasma processes in existing spectral simulation codes and it is the authors' hope that the results shown here will motivate further work in this area.

\section{SUMMARY}

In this article we have explored the two major ways in which spectral lineshapes play a role in the study of high density ICF plasmas. The 
first and most well known use of lineshapes is to provide a means of diagnosing density and temperature in high density implosions. There has been much progress in this area in recent years, primarily due to the advent of generalized line broadening codes which allow line profiles to be computed for complex emitters. As a result the behavior of the line profile of $\mathrm{Ar} \mathrm{He}-\beta$ and its associated dielectronic satellites has been used as a diagnostic of plasma conditions in Nova implosions. In a similar vein, line broadening of 3-2, 4-2, and 5-2 transitions in Ne-like $X e$ has been quantified and shown to be a viable density diagnostic in future high density targets.

The second major role lineshapes play in the study of dense ICF plasmas is in dense plasma spectral simulation. The hundreds to thousands of lineshapes required by these codes must be generated relatively quickly. In addition, the problem of self consistently treating line broadening, kinetics, and general plasma microfield related effects such as continuum lowering in a simulation context remains essentially unsolved. As particular examples relevant to ICF we have shown that line transfer of Stark broadened lines as well as continuum lowering are important. We hope that the demonstration of the importance of these effects to simulation of ICF experiments motivates further work in this very interesting and rich area of physics.

\section{ACKNOWLEDGMENTS}

The authors acknowledge the support of the LLNL ICF program in carrying out this research. This work was performed under the auspices of the U.S. Department of Energy by the Separment of Energy by Lawrence Livermore National Laboratory under contract No. W-7405ENG-48.

\section{REFERENCES}

1. M.H. Key, C.L.S. Lewis, J.G. Lunney, A. Moore, J.M. Ward, and R.K. Thareja, Phys. Rev. Lett. 44, 1669 (1980).

2. C.F. Hooper, Jr., D.P. Kilcrease, R.C. Mancini, L.A. Woltz, D.K. Bradley, P.A. Jaanimagi, and M.C. Richardson, Phys. Rev. Lett. 63,267 (1989).

3. A. Hauer, R.D. Cowan, B. Yaakobi, O. Barnouin, and R. Epstein, Phys. Rev. A 34,411 (1986). 
4. C.J. Keane, R.W. Lee, B.A. Hammel, A.L. Osterheld, L.J. Suter, A. Calisti, F. Khelfaoui, R. Stamm, and B. Talin, Rev. Sci. Inst. 61, 2780 (1990).

5. B.A. Hammel, C.J. Keane, D.R. Kania, J.D. Kilkenny', R.W. Lee, R. Pasha, R.E. Turner, and N.D. Delamater, Rev. Sci. Inst. 63, 5017 (1992).

6. B.A. Hammel, C.J. Keane, D.R. Kania, J.D. Kilkenny, R.W. Lee, R. Pasha, R.E. Turner, and N.D. Delamater, J. Quant. Spec. Radiat. Transfer 51, 113 (1994).

7. C.J. Keane, B.A. Hammel, D.R. Kania, R.W. Lee, A.L. Osterheld, L.J. Suter, R.C. Mancini, C.F. Hooper, Jr., and N.D. Delamater, J. Quant. Spec. Radiat. Transfer 51, 147 (1994).

8. B.A. Hammel, C.J. Keane, M.D. Cable, J.D. Kilkenny, R.W. Lee, and R. Pasha, Phys. Rev. Lett. 70,1263 (1993).

9. C.J. Keane, B.A. Hammel, D.R. Kania, J.D. Kilkenny, R.W. Lee, A.L. Osterheld, L.J. Suter, R.C. Mancini, C.F. Hooper, Jr., and N.D. Delamater, Phys. Fluids B $\underline{5}, 3328$ (1994).

10. H.R. Griem, Phys. Fluids B 42346 (1992).

11. C.J. Keane, B.A. Hammel, A.L. Osterheld, and D.R. Kania, Phys. Rev. Lett. 72,3029 (1994).

12. C.J. Keane, R.W. Lee, and J.P. Grandy, in Proceedings of the 4th International Workshop on the Radiative Properties of Hot Dense Matter, edited by W. Goldstein, C. Hooper, J. Gauthier, J. Seely, and R.W. Lee (Singapore, World Scientific, 1991),p.233.

13. H.A. Scott and R.W. Mayle, Appl. Phys. B 58,35 (1994).

14. O. Peyrusse, J. Quant. Spec. Radiat. Transfer 51 , 281 (1994).

15. R.W. Lee, J.I. Castor, C.A. Iglesias, F.J. Rogers, in Spectral Line Shapes: Vol. 7, (R. Stamm and B. Talin, eds.), Nova Science Publishers, NY, NY (1994).

16. J.I. Castor, P. Dykema, and R.I. Klein, Ap. J. 387, 561 (1992).

17. G. Olson, J. Comly, J.K. La Gattuta, and D.P. Kilcrease, J. Quant. Spec. Radiat. Transfer 51,255 (1994).

18. R.C. Mancini, C.F. Hooper, Jr., N.D. Delamater, A. Hauer, C.J. Keane, B.A. Hammel, and J.K. Nash, Rev. Sci. Inst. 63, 5119 (1992).

19. C.J. Keane, R.C. Cook, T.R. Dittrich, B.A. Hammel, W.K. Levedahl, O.L. Landen, S.H. Langer, D.H. Munro, and H.A. Scott, "Diagnosis of Pusher-Fuel Mix in Spherical Implosions Using X-ray Spectroscopy," to be published in Rev. Sci. Inst.

20. R. Stamm, B. Talin, E.L. Pollock, and C.A. Iglesias, Phys. Rev. A 34, 4144 (1986).

21. L.A. Woltz and C.F. Hooper, Jr., Phys. Rev. A $\underline{38}, 4766$ (1988).

22. R.C. Mancini, D.P. Kilcrease, L.A. Woltz, and C.F. Hooper, Jr., Comp. Phys. Comm. 63, 314 (1991). 
23. N.D. Delamater, G.D. Pollak, A.A. Hauer, R.C. Mancini, C.F. Hooper, Jr., C.J. Keane, B.A. Hammel, and J.K. Nash, Bull. Am. Phys. Soc. 37,1431 (1992).

24. D. A. Haynes, Jr., C.F. Hooper, Jr., R.C. Mancini, D.K. Bradley, J. Delettrez, R. Epstein, and P.A. Jaanimagi, "Spectroscopic Analysis of Ar-doped Laser Driven Implosions," to be published in Rev. Sci. Inst.

25. A. Calisti, L. Godbert, R. Stamm, B. Talin, C.J. Keane, and R.W. Lee, to be submitted to Phys. Rev. E.

26. A. Calisti, L. Godbert, R. Stamm, and B. Talin, J. Quant. Spec. Radiat. Transfer $\underline{51}, 59$ (1994).

27. D.B. Boercker, in Spectral Line Shapes: Vol. 7, (R. Stamm and B. Talin, eds.), Nova Science Publishers, NY, NY (1994).

28. D.B. Boercker, C.A. Iglesias, and J.W. Dufty, Phys. Rev. A $\underline{36} 2254$ (1987).

29. A.E. Koniges, D.C. Eder, A.S. Wan, H.A. Scott, H.E. Dalhed, R.W. Mayle, and D.E. Post, "Role of Radiation in Vapor Shielding of First Wall During Disruption," to be published in J. Nuc. Materials.

30. R.A. London, M.D. Rosen, M.S. Maxon, D.C. Eder, and P.L. Hagelstein, J. Phys. B 22, 3363 (1989).

31. R.M. More, J. Quant. Spec. Rad. Transfer 27345 (1982).

32. H.R. Griem, private communication at Lawrence Livermore National Laboratory, 1994.

33. G.B. Zimmerman and W.L. Kruer, Plasma Phys. 11, 51 (1975).

34. T.R. Dittrich, B.A. Hammel, C.J. Keane, R. McEachern, R.E. Turner, S.W. Haan, and L.J. Suter, "Diagnosis of Pusher-Fuel Mix in Indirectly Driven Implosions," to be published in Phys. Rev. Lett. 